\title{
Phrasing influences the recognition of melodies
}

\author{
PENNY CHIAPPE and MARK A. SCHMUCKLER \\ University of Toronto, Scarborough College, Scarborough, Ontario, Canada
}

\begin{abstract}
One critical step in the processing of complex auditory information (i.e., language and music) involves organizing such information into hierarchical units, such as phrases. In this study, musically trained and untrained listeners' recognition memory for short, naturalistic melodies varying in their phrase structure was tested. For musically trained subjects, memory for information preceding a phrase boundary was disrupted and memory for information subsequent to a phrase boundary was enhanced relative to memory in similar temporal locations for excerpts not containing a phrase boundary. Musically untrained listeners, in contrast, showed no such differences as a function of the phrasing of the melody. These findings conform with previous results in both psycholinguistics and musical cognition and suggest that the phrase serves as a functional unit in musical processing, guiding the parsing of musical sequences during perception, along with the structuring of memory for musical passages.
\end{abstract}

As the most common exemplars of complex auditory information, language and music are often compared. One basic property shared by both involves their hierarchical structure, with both domains containing information existing at a variety of levels. In language, for example, the speech stream can be organized into basic units such as phonemes or into more extended and complex units such as words, and so on, up to relatively largescale units such as phrases and clauses. Correspondingly, a musical sequence can be characterized in terms of short musical events such as tones and chords, somewhat longer events such as melodic, harmonic, and rhythmic fragments, up to larger units such as musical phrases, then musical sections, and so on.

Studies investigating the hierarchical structuring of linguistic and musical passages suggest that a primary unit of cognitive processing is the phrase. One compelling demonstration of the role of phrase structure involves the localization of short noise bursts (clicks) during sentences (Bertelson \& Tisseyre, 1970, 1972; Fodor \& Bever, 1965; Garrett, Bever, \& Fodor, 1966; Ladefoged \& Broadbent, 1960; Reber, 1973; Reber \& Anderson, 1970) or musical passages (Gregory, 1978; Sloboda \& Gregory, 1980; Stoffer, 1985). For example, Fodor and Bever (1965) found that temporal localization of a click occurring during a sentence migrated towards a syntactic boundary. Similarly, research with music has found that the localization of a click migrated towards a phrase boundary during 6-note (Gregory, 1978) or more extended melodies (Sloboda \& Gregory, 1980). Together, these results im-

The research described in this report was supported by a grant from the Natural Sciences and Engineering Research Council of Canada awarded to the second author. The authors would like to thank two anonymous reviewers for their comments concerning a previous version of this manuscript. Correspondence concerning this paper can be addressed to M. A. Schmuckler, Division of Life Sciences, University of Toronto, Scarborough College, Scarborough, ON, Canada MIC 1A4 (e-mail: marksch@lake.scar.utoronto.ca). plicate the phrase as a primary, functional unit in the processing of language and music.

Given its importance in perception, it is reasonable to anticipate that phrase structure will play a role in memory for speech and music. Tests of memory for linguistic materials (Caplan, 1972; Jarvella, 1970, 1971) have, in fact, demonstrated that the interposition of a phrase or clause boundary in text disrupts short-term memory for information preceding, but not following, this boundary. Jarvella $(1970,1971)$, for example, found that recall of the last seven words of connected discourse prior to a pause was better when these words occurred in the same clause than when these words straddled a clause boundary. Jarvella (1971) interpreted this result as suggesting that the clause functions as a semantic processing unit.

Research in musical cognition has also demonstrated that syntactic structure influences memory for musical materials. Tan, Aiello, and Bever (1981), for example, investigated the impact of melodic phrase structure on musical memory. In this work, musically trained and musically untrained listeners heard two-phrase melodies consisting of simplistic, abstract musical patterns (e.g., equitemporal arpeggiations of musical chords). Following these melodies, listeners determined whether a 2-note probe had occurred in the preceding melody. The probe was taken from one of three positions-the 2 notes preceding or following the phrase boundary ("withinphrase" probes) and the 2 notes straddling the phrase boundary ("between-phrase" probes). Supporting the idea that musical memory is influenced by phrase structure, recognition was worse for between-phrase than for within-phrase probes.

The current study replicates and extends this work in a number of ways. First, to generalize Tan et al.'s results to a naturalistic context, this study used melodies drawn from the corpus of Western tonal music, as opposed to the schematic melodies employed by these earlier researchers. In musical cognition research, there exists a continual tension between employing, as stimuli, idealized musical 
materials that simplify the musical relationships occurring in real music and naturalistic musical passages that are difficult to quantify and potentially idiosyncratic to a given composer or style (Krumhansl, 1990). Given this tension it is important to demonstrate that the cognitive processes identified using simplified, abstract materials operate in more natural musical contexts.

Second, this study explored the import of different musical cues for phrase boundaries than had been previously examined. In Tan et al.'s (1981) melodies, phrase boundaries were marked through the sole cue of a change in the harmonic structure underlying the melodies. In the current study, the phrase structure of the naturalistic musical passages was determined intuitively, on the basis of such cues as pitch discontinuities (frequency differences between adjacent notes crossing a phrase boundary) and rhythmic variations (duration differences between adjacent notes crossing a phrase boundary). Krumhansl and Jusczyk (1990; Jusczyk \& Krumhansl, 1993) have observed that such cues characterize naturally (but not unnaturally) segmented phrases of Mozart minuets.

Third, this study combined methodological aspects of Tan et al. (1981) and Jarvella $(1970,1971)$ in looking at recognition memory for long melodic fragments, relative to Tan et al. (1981). Specifically, listeners heard a melody followed by a 7-note probe, and judged whether this probe was identical to the final 7 notes of the earlier melody. The 7-note probes either were all part of a single phrase or they crossed a phrase boundary. On the basis of Jarvella's $(1970,1971)$ and Tan et al.'s (1981) work, we would expect that memory for the different notes of the 7-note probe would vary with the phrasing of the melody.

Finally, this experiment assessed the role of musical training in recognition memory for such melodies. Tan et al. (1981) found that, whereas musical training did not vary the impact of phrasing on recognition memory for strongly marked musical phrases, weaker markers of phrase structure produced memory differences among within- and between-phrase probes only for musically sophisticated listeners. Given that, in the current study, phrase boundaries were marked with multiple rather than single cues, one might predict that memory will not vary with musical training. On the other hand, the melodies of this study were more musically complex than Tan et al.'s stimuli and employed longer recognition probes. Accordingly, musical training might afford an advantage in recognizing phrase structure, restricting differences in memory for between- and within-phrase melodies to musically sophisticated listeners.

\section{METHOD}

\section{Subjects}

Eighteen students from the University of Toronto, who received course credit in introductory psychology or $\$ 7$ as compensation, participated in this study. Nine listeners, who had had a minimum of 3 years of musical instruction, were classified as trained musicians, having received a mean of 8.2 years of musical training and currently being involved in musical activities for approximately $3.1 \mathrm{~h}$ per week.
The remaining subjects were classified as untrained, having received 0.2 years of musical training and currently being involved in musicmaking activities for $0.7 \mathrm{~h}$ per week.

\section{Apparatus}

All stimuli were generated by a Yamaha TX816 synthesizer, controlled by a IBM-PC-compatible microcomputer, using a Roland MPU-401 MIDI interface. Sounds were mixed, using a Mackie 1202 mixer, and amplified and presented to the listeners through a Boss MA-12 speaker. The timbre of all stimuli approximated that of a piano (see Schmuckler, 1989).

\section{Stimulus Materials}

Trials consisted of a standard melody followed by a probe excerpt. Thirty-four Schumann songs were (with minor adaptations) chosen as the standard melodies; none of these melodies were recognized by the listeners. Two standard versions of each melody were created, with each having the same starting note but ending at a different location. For a "within-phrase" standard, the last 7 notes all lay within a single phrase. In a "between-phrase" standard, the last 7 notes crossed a phrase boundary, with either the final 3 (or 4 ) notes of the melody drawn from a phrase that was different from the preceding notes. Seventeen of the within-phrase standards were shorter than their matched betweenphrase standard, whereas the remaining 17 within-phrase standards were longer than their between-phrase standard.

Three different probe excerpts, based on the last 7 notes of the standard melody, were created for each standard. The first of these was an "unchanged" probe, and was identical to the last 7 notes of the standard. The other two probes were "changed" probes, with one of the notes altered relative to the standard. In half of these excerpts, the 2 nd or 3rd note was changed; these will be referred to as "early" changes. In the remaining changed excerpts, the 4 th, 5 th, or 6 th note was changed; these will be referred to as "late" changes. The substituted (i.e., changed) notes were chosen to remain within the overall tonality of the passages, and to not drastically alter the global contour of the original melody. Figure 1 shows a sample between- and withinphrase standard melody and its related probes.

All subjects heard two blocks of 136 trials, with each block consisting of 68 standard-plus-unchanged probe trials and 68 standardplus-changed probe trials. Within each block, the 68 standard-plusunchanged probe trials consisted of 17 between-phrase standards and 17 within-phrase standards (two repetitions each). The 68 standardplus-changed probe trials consisted of the early and late changed versions of the between- and within-phrase standards. The other block of trials consisted of the complementary set of between- and withinphrase trials. Order of presentation of the two blocks was counterbalanced across listeners, and the order of trials within each block was randomized for each listener.

\section{Procedure}

The listeners were informed that they would hear a series of pairs of short melodic fragments, with each pair containing a standard melody followed 1 sec later by a 7 -note probe. Their task was to decide if the probe matched the last 7 notes of the standard melody and, if so, to respond SAME by pressing the " $l$ " key and, if not, to respond DIFFERENT by pressing the "0" key on the computer keyboard. Once the listener had responded, the next trial began automatically. After the experimental trials, the listeners completed a musical background questionnaire and were debriefed as to the purposes of this study. The entire session lasted approximately $1^{1 / 4} \mathrm{~h}$.

\section{RESULTS}

For each listener, correct recognition for the withinand between-phrase standards for both early and late changes was aggregated across the 34 melodies, and $d^{\prime} \mathrm{s}$ were computed using the percent correct for the standardplus-unchanged probe trials as the hit rate and the per- 


\section{Between-phrase}

Standard
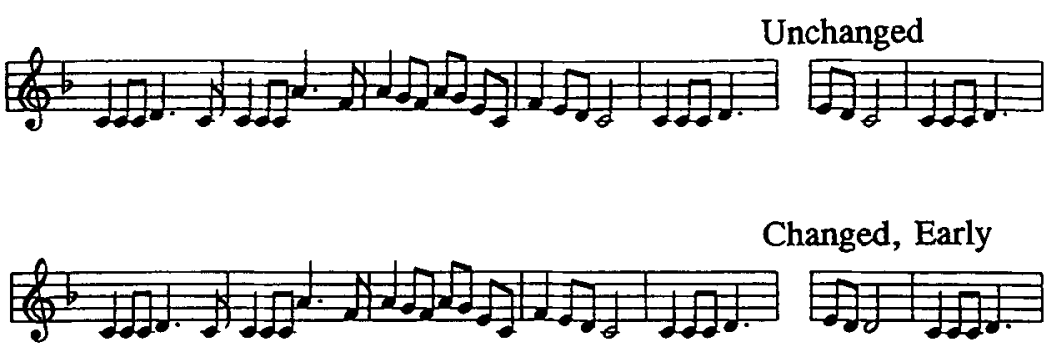

Changed, Early

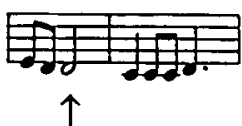

Changed, Late
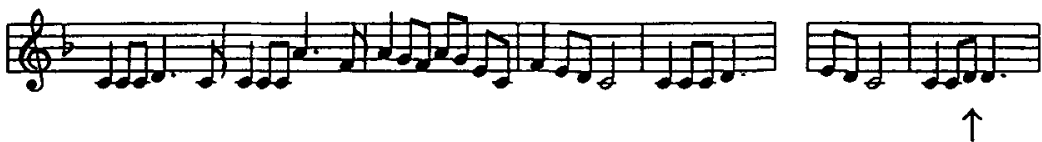

\section{Within-phrase}

Standard
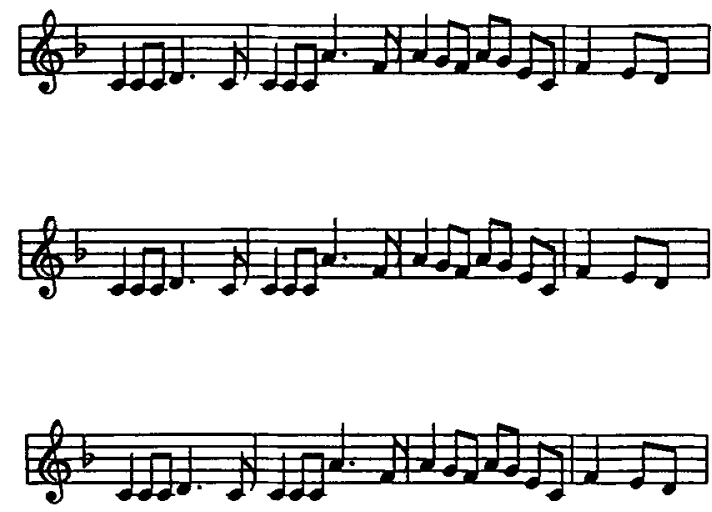

Probe:

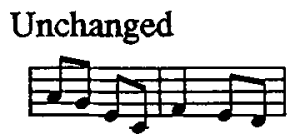

Changed, Early

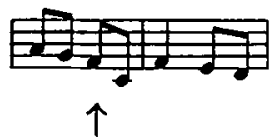

Changed, Late

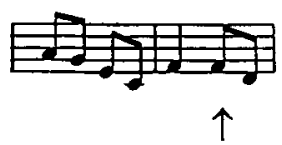

Figure 1. Sample between- and within-phrase stimuli used in this experiment. Each standard between-phrase and within-phrase melody is shown with its associated nochange, early-change, and late-change probe. The location of the changed tone is notated with an arrow.

cent incorrect for the standard-plus-changed probe trials as the false-alarm rate. To avoid the problem of infinite $d^{\prime}$ s $(100 \%$ or $0 \%$ hit or false-alarm rate), which were assumed to reflect sampling error, hit and false-alarm rates were adjusted by adding .5 to the number correct and dividing this number by the total number of trials plus 1 . This manipulation has little effect on the proportions and relative ranking of $d^{\prime}$ scores (Thorpe, Trehub, Morrongiello, \& Bull, 1988; Trainor \& Trehub, 1993).

In a four-way analysis of variance (ANOVA), $d$ 's were examined with the within-subjects variables of phrasing (within- vs. between-phrase), change location (early vs. late), and block (Block 1 vs. Block 2), and the betweensubject variable of training (trained vs. untrained). This analysis revealed a main effect of training $[F(1,16)=$
$4.61, M S_{\mathrm{e}}=2.58, p<.05$ ], with trained listeners outperforming untrained listeners $(M \mathrm{~s}=1.40$ vs. 0.83 , $S D s=0.83$ vs. 0.76 , respectively), and a main effect of change location $\left[F(1,16)=65.46, M S_{\mathrm{e}}=0.23, p<.001\right.$; $M \mathrm{~s}=0.79$ and $1.44, S D \mathrm{~s}=0.63$ and 0.81 , for early and late changes, respectively]. There was no main effect for block $\left[F(1,16)=1.54, M S_{\mathrm{e}}=0.22\right.$, n.s. $]$ or for phrasing $\left[F(1,16)=0.65, M S_{\mathrm{e}}=0.30\right.$, n.s. $]$. The two-way interaction between block and phrasing was significant $\left[F(1,16)=5.84, M S_{\mathrm{e}}=0.09, p<.05\right]$, as was the threeway interaction between training, block, and phrasing $\left[F(1,16)=15.17, M S_{\mathrm{e}}=0.09, p<.01\right]$.

The most important result, however, was the significant three-way interaction between phrasing, change location, and musical training $\left[F(1,16)=4.57, M S_{\mathrm{e}}=\right.$ 


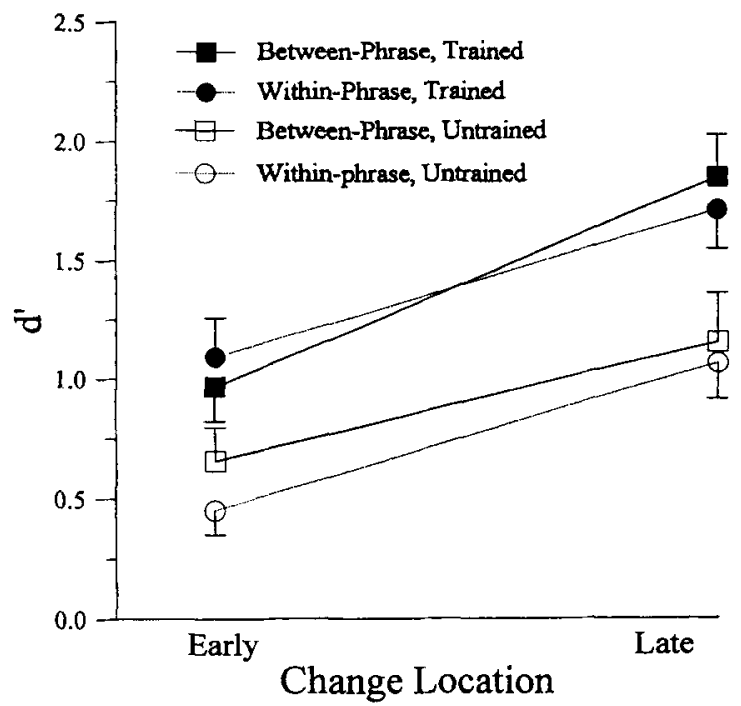

Figure 2. Mean $d$ 's for the detection of early and late change probes for between-phrase and within-phrase melodies for musically trained and musically untrained listeners.

$0.07, p<.05]$, suggesting that the effect of phrasing and change location varied with musical training. Figure 2 graphs the mean $d^{\prime}$ s as a function of change location and phrasing for the musically trained and untrained listeners. Given that block did not generally influence $d$ 's, all subsequent analyses collapsed across this variable.

A pair of subsequent two-way ANOVAs compared $d$ 's as a function of phrasing and change location for the musically trained and untrained listeners individually. For the trained listeners, there were significantly higher $d$ 's for late changes $(M=1.78, S D=0.72)$ relative to early changes $(M=1.03, S D=0.65)\left[F(1,8)=52.73, M S_{\mathrm{e}}=\right.$ $0.10, p<.001]$. The main effect for phrasing was not significant $\left[F(1,8)=0.002, M S_{\mathrm{e}}=0.15, \mathrm{n} . \mathrm{s}\right.$.]. The critical result, however, was the significant interaction between phrasing and change location $[F(1,8)=6.70$, $\left.M S_{\mathrm{e}}=0.02, p<.05\right]$, with the difference between recognition memory for early versus late changes more pronounced for between-phrase melodies, relative to withinphrase melodies (see Figure 2).

For the untrained listeners there was again significantly better memory for late changes $(M=1.10, S D=$ $0.76)$ compared with early changes $(M=0.55, S D=$ $0.52)\left[F(1,8)=19.96, M S_{\mathrm{e}}=0.14, p<.005\right]$. Again, there was no effect of phrasing $\left[F(1,8)=1.24, M S_{\mathrm{e}}=\right.$ 0.14 , n.s.]. Most interestingly, and in contrast to the trained listeners, phrasing did not differentially influence recognition memory for early versus late changes $[F(1,8)$ $=0.68, M S_{\mathrm{e}}=0.05$, n.s.] (see Figure 2).

\section{DISCUSSION}

Generally, these findings validate Tan et al. (1981) and generalize the results of Jarvella $(1970,1971)$ to musical stimuli. One of the strongest findings of this work was a recency effect, with listeners showing enhanced recognition for more recent information than for material presented earlier. Superimposed onto this recency effect (not in itself especially noteworthy) was the finding that, for musically trained listeners, recognition of early versus late changes in a 7-note melodic fragment was influenced by the phrase structure of the original melody. For fragments crossing a phrase boundary, memory for the earlier phrase was reduced and memory for the latter phrase enhanced relative to when fragments did not contain a phrase boundary. In contrast, musically untrained listeners were not influenced by the phrasing of melodies, although they were by the recency of these changes.

The finding that the musically trained listeners' recognition memory for melodies was influenced by phrasing echoes results found in psycholinguistics (e.g., Jarvella, 1970, 1971), suggesting that musical phrases are distinct functional units during musical processing. Accordingly, in between-phrase melodies, early information, which is part of a completed unit, is removed from auditory store, resulting in poor memory for this information. Correspondingly, information occurring in the second phrase is well remembered, given the increased resources available for its processing. In contrast, for within-phrase melodies, because all the information from the probe notes is retained in auditory store, the difference in memory for early versus late information is reduced relative to between-phrase melodies, although there is still a recency effect.

Although these findings are not surprising given previous results implicating the phrase as a critical unit of musical processing, they do extend this earlier work in important ways. One of the most significant extensions offered by this work involves the demonstration that melodic phrasing impacts on recognition memory when employing as stimuli naturalistic melodic materials. Given the previously discussed tension in musical cognition between choosing artificial yet well-controlled music versus naturalistic, potentially idiosyncratic music as stimuli, it is both critical (and reassuring) to observe similar cognitive processes operating in perception and memory for both subsets of materials. Together, the results of Tan et al. (1981) and the current study provide just this situation.

This study also extends earlier findings in terms of the cues used to mark phrase boundaries. In Tan et al. (1981), phrases were marked by implied changes in harmony. In this study, however, a post hoc examination of these melodies revealed other cues demarcating phrase boundaries. Specifically, the melodies were examined for cues for phrasing on the basis of the findings of Krumhansl and Jusczyk ( 1990 ; Jusczyk \& Krumhansl, 1993). Those authors observed a sharp drop in the average pitch height of tones leading up to the phrase boundary, with a corresponding jump in pitch height immediately after the phrase boundary. Along with such pitch variation was a sharp increase in pitch duration before the phrase boundary, with a corresponding drop in duration subsequent to the phrase boundary (see Krumhansl \& Jusczyk, 1990, Figure 2).

To assess the phrasing of the stimuli from the present study, the three tones prior to the phrase boundary of the standard, betweenphrase melodies were compared with the three tones subsequent to the phrase boundary; the standard, within-phrase melodies were similarly split on the basis of the between-phrase structure for that same melody. ${ }^{2}$ To analyze pitch height, the tone immediately preceding the phrase boundary was given the value of 0 , and the distance between this tone and the other tones, in semitone steps (the smallest pitch distance employed by Western tonal music), was counted. The top half of Figure 3 shows the average pitch changes for the between- and withinphrase melodies. Mirroring Krumhansl and Jusczyk's (1990) findings, the between-phrase melodies produced a drop in pitch height prior to the phrase boundary and an increase in pitch height following this boundary. In contrast, the within-phrase melodies contained no dramatic decrease in pitch height prior to the phrase boundary and no change in pitch height subsequent to this boundary.

To analyze pitch duration, the tone immediately preceding the phrase boundary was assigned a value of 4 , with prior and subsequent notes assigned durations relative to that note. The bottom half of Figure 3 shows the average duration changes for the between- and withinphrase melodies. Again, mirroring Krumhansl and Jusczyk (1990), phrase boundaries for the between-phrase melodies were marked by an increase in duration before the phrase boundary and a decrease in 

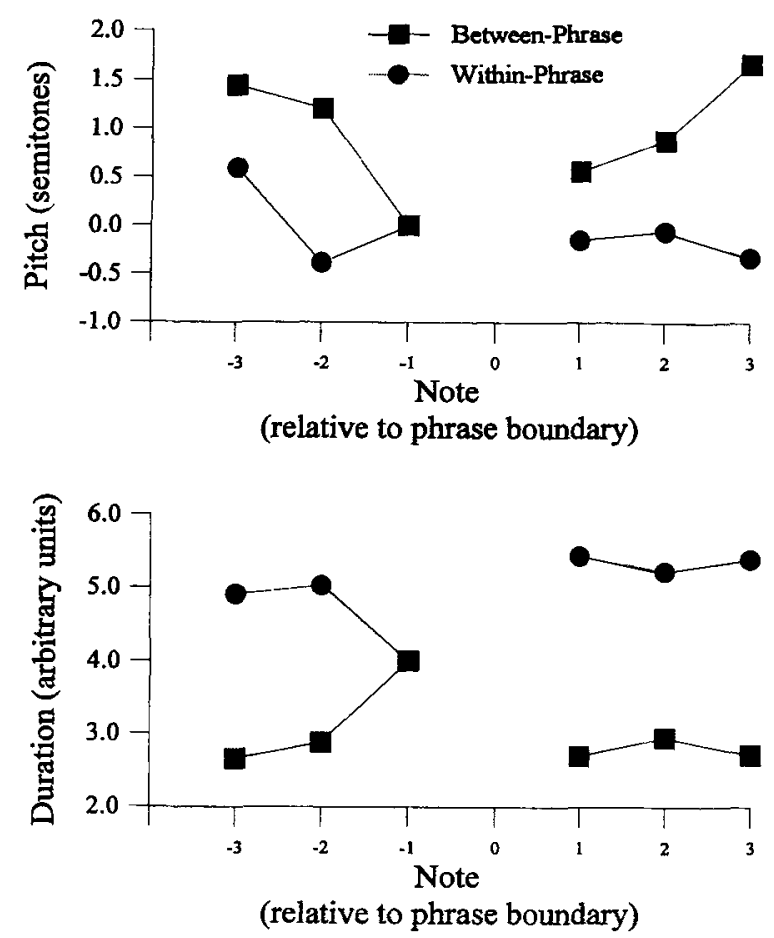

Figure 3. Mean normalized pitch and durations for the 3 notes preceding and following the phrase boundary for the betweenand within-phrase melodies. The phrase boundary is designated as note 0 .

duration following this boundary. This pattern did not characterize the within-phrase melodies, however, which showed a decrease in duration before the phrase boundary, followed by an increase in duration.

Overall, phrase boundaries were marked by systematic variations in pitch contour and note duration around phrase boundaries. Unfortunately, attempts to directly relate the strength of such cues to memory performance are hampered by the fact that the experimental paradigm presented two melodies on each trial (standard and probe) with different probe variants, making it difficult to quantify the available stimulus information of a single trial (although it is possible for the standard melodies alone) and similarly problematic to represent memory performance with a single value. Future research might examine this possibility, systematically varying the phrase boundary cues and exploring the impact of such manipulations on musical memory.

This study also found a consistent difference between musically trained and untrained listeners, with only the trained listeners influenced by the musical phrasing of the melodies. One interpretation of this result is that the phrase cues for these stimuli were simply too subtle for untrained listeners, causing them to miss the implied phrase structure of the melodies. Unfortunately, this interpretation is difficult to reconcile with results suggesting that young infants are sensitive to just this type of information for phrase structure in musical passages (Jusczyk \& Krumhansl, 1993; Krumhansl \& Jusczyk, 1990).

There are problems, however, in comparing the two situations. Ignoring, for the moment, the obvious difficulties in comparing infant and adult performance on any task, Krumhansl and Jusczyk's (1990) studies examined infant preferences for musical passages, whereas the current study examined recognition memory; this basic difference could produce a divergence in results. Second, Krumhansl and Jusczyk compared preferential listening to naturally versus unnaturally segmented musical passages, whereas this study employed two versions of well-formed melodies. Had this study employed musical passages varying in the naturalness of their phrase structure, such differences might well have been reflected in recognition memory by untrained listeners. Finally, although the cues for phrasing were comparable to those identified by Krumhansl and Jusczyk, the magnitudes of the differences were much less, with, for example, pitch variation around the phrase boundary only about 1 to 2 semitones (as compared with over 12 semitones in Krumhansl \& Jusczyk, 1990). As such, all these differences make it difficult to compare the current situation with the research with infants.

The (relatively) small differences in pitch height at phrase boundaries, along with the absence of other potent cues for phrasing such as harmonic closure, might well have rendered the phrase structure of these melodies discernible only to musically trained listeners. If true, then the differences in recognition memory as a function of training actually concur with Tan et al. (1981), who found that musically unsophisticated listeners' memory was influenced only by strongly marked musical phrases. Given the nature of these phrasing cues, along with the longer, more complex recognition probes, musical training could well have afforded distinct processing advantages. In fact, the observed difference between trained and untrained listeners in overall memory performance is suggestive of just such a processing difference. This question could similarly be examined in future research by testing both musically sophisticated and musically unsophisticated listeners with musical passages in which the cues for phrase structure vary from subtle to strong.

In sum, the current experiment demonstrated that phrase structure plays a significant role in the cognitive processing of musical sequences. In this sense, the phrase serves as a functional unit, guiding the processing of musical sequences during perception, and the structuring of memory for musical passages. Further work on the relation between perception and/or memory for music and surface phrase structure will garner insights into the processes involved in musical cognition, and potentially the apprehension of complex auditory information more generally.

\section{REFERENCES}

Bertelson, P., \& Tisseyre, F. (1970). Perceiving the sequence of speech and non-speech. Quarterly Journal of Experimental Psychology, 22, 656-662.

BerTelson, P., \& TISSEYRE, F. (1972). Lateral asymmetry in the perceived sequence of speech and nonspeech stimuli. Perception \& Psychophysics, 11, 356-362.

CaPlan, D. (1972). Clause boundaries and recognition latencies for words in sentences. Perception \& Psychophysics, 12, 73-76.

Fodor, J. A., \& Bever, T. G. (1965). The psychological reality of linguistic segments. Journal of Verbal Learning \& Verbal Behavior, $\mathbf{4}$, 4\4-420.

Garrett, M., Bever, T. G., \& Fodor, J. A. (1966). The active use of grammar in speech perception. Perception \& Psychophysics, 1, 3032 .

Gregory, A. H. (1978). Perception of elicks in music. Perception \& Psychophysics, 24, 171-174.

JARVELLA, R. J. (1970). Effects of syntax on running memory span for connected discourse. Psychonomic Science, 19, 235-236.

JARVELLA, R. J. (1971). Syntactic processing of connected speech. Journal of Verbal Learning \& Verbal Behavior, 10, 409-416.

JusczyK, P. W., \& KrumhansL, C. L. (1993). Pitch and rhythmic patterns affecting infants' sensitivity to musical phrase structure. Journal of Experimental Psychology: Human Perception \& Performance, 19, 627-640.

Krumhansl, C. L. (1990). Cognitive foundations of musical pitch. London: Oxford University Press.

Krumhansl, C. L., \& JusczYK, P. W. (1990). Infants' perception of phrase structure in music. Psychological Science, 1, 70-73.

Krumhansl, C. L., \& SChMuCKLER, M. A. (1986). Key-finding in music: An algorithm based on pattern matching to tonal hierarchies. Paper presented at the 19th Annual Mathematical Psychology Meeting, Cambridge, MA.

Ladefoged, P., \& Broadbent, D. E. (1960). Perception of sequence in auditory events. Quarterly Journal of Experimental Psychology, 12, $162-170$

REBER, A. S. (1973). Locating clicks in sentences: Left, center, and right. Perception \& Psychophysics, 13, 133-138. 
REBER, A. S., \& ANDERSON, J. R. (1970). The perception of clicks in linguistic and nonlinguistic messages. Perception \& Psychophysics, 8, 81-89.

SCHMUCKLER, M. A. (1989). Expectation in music: Investigation of melodic and harmonic processes. Music Perception, 7, 109-150.

Sloboda, J. A., \& GREgory, A. H. (1980). The psychological reality of musical segments. Canadian Journal of Psychology, 34, 274-280.

STOFFER, T. H. (1985). Representation of phrase structure in the perception of music. Music Perception, 2, 191-220.

TAN, N., Aiello, R., \& BeVER, T. G. (1981). Harmonic structure as a determinant of melodic organization. Memory \& Cognition, 9, 533-539.

Thorpe, L. A., Trehub, S. E., Morrongiello, B. A., \& Bull, D. (1988). Perceptual grouping by infants and preschool children. $\mathrm{De}$ velopmental Psychologv, 24, 484-491

Trainor, L. J., \& Trehub, S. E. (1993). Musical context effects in infants and adults: Key distance. Journal of Experimental Psychology: Human Perception \& Performance, 19, 615-626.

\section{NOTES}

1. Although differences in recognition have been attributed to the impact of phrasing, it is possible that other factors varied systematically in these melodies. One possibility is the amount of time between the occurrence of the changed note and the subsequent response to the melody, with longer delays leading to worse memory. This possibility was tested by comparing the absolute time (in milliseconds), the num- ber of beats, and the number of notes between the changed note and the subsequent end of the probe as a function of phrasing and change location. None of these analyses revealed an interaction between these variables, although there were (obviously) main effects for change location.

A subsequent series of analyses examined whether the changed note systematically altered the tonal implications of these melodies. Musical tonality, which refers to the structuring of the tones of the chromatic scale around a central, reference pitch, plays a critical role in the perception and memory of musical passages (see Krumhansl, 1990). Tonality was assessed by calculating the implied tonal structure of all of the variants of these melodies, and computing the distance in tonal space between the unchanged and the changed versions, using the key-finding algorithm of Krumhansl and Schmuckler (1986) described in Krumhansl (1990). These analyses failed to undercover any systematic differences in tonal space as a function of phrasing or change-location variables, suggesting that tonality had little impact in the current situation.

2. The astute reader will note that this analysis uses only 6 of the 7 probe notes. Analyses were restricted to this subset because only 13 of the 34 melodies contained 4 notes prior to the phrase boundary. Accordingly, analyses based on the 4 th note prior to the phrase boundary were felt to be somewhat unreliable.

(Manuscript received April 29, 1996; revision accepted for publication September 18, 1996.) 